\title{
Penetration of the Earth's free oscillations at 54 minute period into the atmosphere
}

\author{
G. M. Shved, L. N. Petrova, O. S. Polyakova \\ Departments of Atmospheric Physics and Earth Physics, Institute of Physics, St. Petersburg State University, \\ St. Petersburg-Petrodvorets 198904, Russia
}

Received: 12 May 1999 / Revised: 2 February 2000 / Accepted: 16 February 2000

\begin{abstract}
It is known that the fundamental spheroidal mode ${ }_{0} S_{2}$ of the Earth free oscillation with a period of about $54 \mathrm{~min}$ forces atmospheric oscillations. We present a certain phase relationship for components of the ${ }_{0} S_{2}$ multiplet, which is based on synchronous collocated microbarograph and seismograph observations. This relationship is both the first observational manifestation of the Pekeris mode of global atmospheric oscillations with the $54 \mathrm{~min}$ period and a further proof of the Earth's ${ }_{0} S_{2}$ mode penetrating into the atmosphere. We show that the linear non-dissipative model of steady forced oscillations in isothermal atmosphere at rest does not describe the penetration of the ${ }_{0} S_{2}$ mode into the atmosphere adequately.
\end{abstract}

Key words: Meteorology and atmospheric dynamics (middle atmosphere dynamics; waves and tides).

\section{Introduction}

There are Earth free oscillations observed with seismographs (e.g. Aki and Richards, 1980). The lowest frequency mode of the oscillations is the fundamental spheroidal mode ${ }_{0} S_{2}$ that has a period of about $54 \mathrm{~min}$. The Earth's departure from spherical symmetry such as diurnal rotation, ellipticity, and lateral heterogeneity splits the mode ${ }_{0} S_{2}$ into five components spaced about $50 \mathrm{~s}$ apart. The first observations of these components occurred after very large earthquakes with a magnitude M $>7$ (e.g. Buland et al., 1979; Aki and Richards, 1980). However, subsequently they were also observed on seismically quiet days (e.g. Lin'kov et al., 1991), much like the Earth free oscillations ${ }_{0} S_{n}$ with $n \approx 15-65$

Correspondence to: G. M. Shved

e-mail: shved@lmupa.phys.spbu.ru of the 2-8 min period range (Suda et al., 1998; Tanimoto et al., 1998).

Oscillations with a period of about $54 \mathrm{~min}$ and even the splitting inherent in the ${ }_{0} S_{2}$ mode have been observed in geomagnetic disturbances (Roldugin, 1967; Bobova et al., 1990). The amplitude of these oscillations is intensified during global seismic activities. The components of the ${ }_{0} S_{2}$ mode have also been detected with microbarograph in ground pressure variations (Garmash et al., 1989; Lin'kov et al., 1991). There are microbarographic observations of the ${ }_{0} S_{2}$ oscillations penetrating into the atmosphere at a seismically inactive period.

This work constitutes a further observational study of forcing atmospheric oscillations by the Earth's ${ }_{0} S_{2}$ oscillations. We use synchronous collocated measurements by a microbarograph and seismograph to determine a phase difference between the seismic oscillations and the pressure ones for the different components of the ${ }_{0} S_{2}$ mode. By comparing these phase differences, we first reveal experimentally the oscillation frequency corresponding to the Pekeris solution in the global oscillation theory for the Earth's atmosphere (Dikii, 1965). We also discuss mechanisms of the ${ }_{0} S_{2}$ mode penetrating into the atmosphere.

\section{Instrumentation}

The ground pressure $(p)$ variations have been measured with a microbarograph, made on the basis of the standard millibarograph M-22 (Kozhevnikova et al., 1980). As far as the seismograph is concerned, Kirnos' vertical pendulum (Savarensky and Kirnos, 1955), protected from the direct effect of variations of the atmospheric pressure and temperature with a special chamber (Lin'kov et al., 1982), has been used. The sensors of both instruments are provided with converters which contain photodiodes. A set of filters, employed at the output of the converters, has resulted in the detection of oscillations with period $(\tau)$ in the $\sim 0.5-5 \mathrm{~h}$ 
range. For this $\tau$ range the seismograph records vertical acceleration due to a joint action of ground displacement and gravitational acceleration perturbation. The microbarograph sensitivity at the converter output was about $1-5 \mathrm{mWPa}^{-1}$, which has enabled us to measure pressure variations as small as some tenths of a $\mu$ bar. Amplification of the seismometric channel at $\tau=1 \mathrm{~h}$ equals about 10 .

\section{Determination of phase difference}

A spheroidal mode of the Earth free oscillation is symbolized by ${ }_{k} S_{n}$ (e.g. Buland et al., 1979; Aki and Richards, 1980). Here $k$ is the number of nodes between the surface and center of the Earth for the radial wave function of the mode. $k=0$ gives the fundamental mode. The integer $n$ is the degree of the spherical surface functions which describe the horizontal structure of motions associated with the mode. Namely, the radial (vertical) displacement for the $m$ component of the ${ }_{k} S_{n}$ mode splitting is proportional to the complex spherical harmonic $Y_{n}^{m}(\vartheta, \varphi)$ where $\vartheta$ is the co-latitude and $\varphi$ is the longitude, $-n \leq m \leq n$. So, the ${ }_{k} S_{n}$ mode is split into $2 n+1$ components (lines).

The frequencies of the ${ }_{0} S_{2}$ mode components are $0.3000, \quad 0.3048, \quad 0.3095, \quad 0.3140, \quad 0.3185 \mathrm{mHz}$ for $m=-2,-1,0,1,2$, respectively (Buland et al., 1979). The analysis of seismograph records, as a rule, makes possible to detect only some of the five components (e.g., Lin'kov et al., 1991). The wide scatter of relative powers of the components in the concrete records results mainly from interference between the ${ }_{0} S_{2}$ multiplet components due to the small frequency spacing (e.g. Aki and Richards, 1980). It is established for the ${ }_{0} S_{2}$ oscillations forced by a well-identified earthquake that the observed distribution of the Earth ${ }_{0} S_{2}$ components over power depends on latitude and longitude of both a measurement site and earthquake source as well as on other parameters of the earthquake (e.g., Mendiguren, 1973; Stein and Geller, 1977). As for detecting the ${ }_{0} S_{2}$ components with microbarographic measurements, the gravity waves of atmospheric sources can hinder detection of these components. Moreover, it may turn out that some of the components are not detected due to a strong frequency dependence of the efficiency of oscillation transmission into the atmosphere (see Sect. 5).

Our goal is to compare the phase differences $(\Delta \psi)$ between seismic and atmospheric oscillations for the ${ }_{0} S_{2}$ components disposed on different sides of the central component $(m=0)$ along the frequency axis. We do succeed in finding three synchronous collocated microbarographic and seismographic runs to compare $\Delta \psi_{m}$ for $m>0$ and $m<0$ (Table 1). The sampling time of measurements in $4 \mathrm{~min}$. As an example we show time series (Fig. 1) and their power and coherence spectra (Figs. 2 and 3) for one of the three cases. It can be seen (Fig. 2) that the power spectra of seismic oscillations and ground pressure variations are significantly distinguished. However, the spectra exhibit common harmonics near the ${ }_{0} S_{2}$ multiplet frequencies. Figure 3 demonstrates that the correlation between these harmonics of seismic and pressure spectra is statistically justified.

The achievement of the goal in hand is complicated by the facts that (1) the ${ }_{0} S_{2}$ oscillations can be extremely unsteady and (2) there is a need to separate the ${ }_{0} S_{2}$ multiplet components closely spaced in frequency. The conventional fast Fourier transform is not suited to solve this problem. Thus, we have used the method of record processing, described by Savina et al. (1985). First the raw data have been transformed to time series $F\left(t_{i}\right)$, where $t$ is the time, employing a numerical filter with bandpass around the ${ }_{0} S_{2}$ multiplet frequencies. In addition $F(t)$ has been approximated by

$F(t)=\sum_{j}\left(A_{j} \cos \sigma_{j} t+B_{j} \sin \sigma_{j} t\right)+F^{*}(t)$

over the whole time interval of measurements, where $A_{j}, B_{j}$, and $\sigma_{j}$ are the parameters of a $j$-harmonic and $F^{*}(t)$ is the residual "noise" function. The parameters were fitted in a least square sense by using the following procedure. The power spectrum of $F(t)$ gives the initial values of the parameters and the initial number of harmonics used in the fitting. In other words, the harmonics having amplitudes in excess of $1 / 3$ of the largest amplitude are taken. The parameters are sequentially determined in decreasing order of the

Table 1. Comparison of the phase differences between oscillations in the Earth and atmosphere for the components of the Earth's ${ }_{0} S_{2}$ multiplet

\begin{tabular}{|c|c|c|c|c|c|c|}
\hline 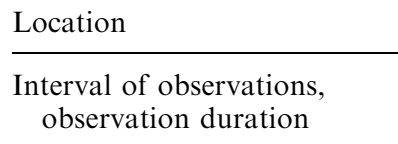 & \multicolumn{4}{|c|}{ St. Petersburg $\left(60^{\circ} \mathrm{N}, 30^{\circ} \mathrm{E}\right)$} & \multicolumn{2}{|c|}{ Tbilisi $\left(42^{\circ} \mathrm{N}, 45^{\circ} \mathrm{E}\right)$} \\
\hline $\begin{array}{l}\text { Phase difference, } \Delta \psi_{m}, \\
\text { between the ground } \\
\text { pressure oscillations } \\
\text { and the seismic oscillations }\end{array}$ & 1.33 & -2.00 & -1.09 & 1.42 & -3.01 & 0.39 \\
\hline$\left|\Delta \psi_{m>0}-\Delta \psi_{m<0}\right|$ & 3.33 & & 2.51 & & 3.40 & \\
\hline
\end{tabular}




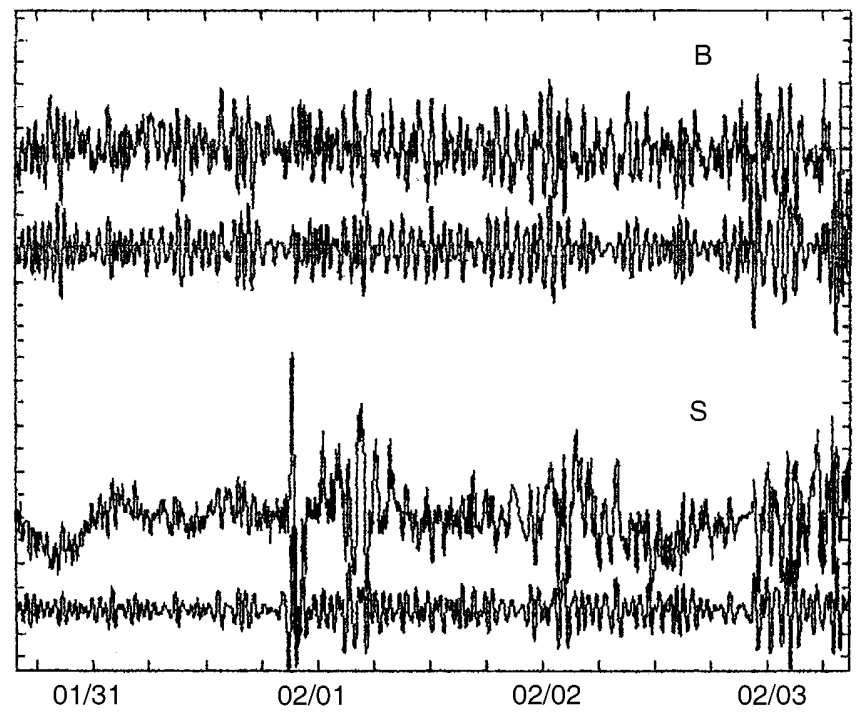

Fig. 1. Synchronous microbarographic $(B)$ and seismographic $(S)$ records during 31 January-3 February 1982 at St. Petersburg $\left(60^{\circ} \mathrm{N}\right.$, $30^{\circ} \mathrm{E}$ ), in relative units. The upper time series are raw data. The lower time series have been derived from the upper ones by eliminating longperiod trends using a smoothing filter

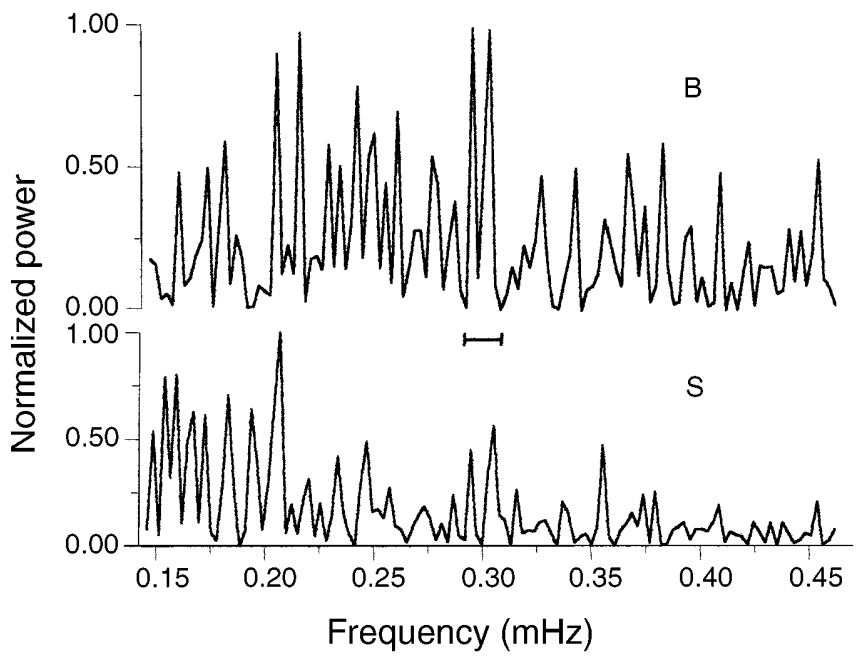

Fig. 2. Power spectra of the unfiltered microbarographic $(B)$ and seismographic $(S)$ time series shown in Fig. 1. Two prominent peaks in both spectra, attributed to the ${ }_{0} S_{2}$ multiplet components, are marked off by a bar

amplitude of harmonic. Those for each harmonic are evaluated from time series derived by eliminating the contributions to $F(t)$ of harmonics with larger amplitudes that the amplitude of a harmonic considered. This special feature of the procedure makes it possible to detect harmonics unresolved by a conventional spectral analysis. This is particularly important for the closely spaced ${ }_{0} S_{2}$ components which interfere and can manifest themselves in a power spectrum as a single wide asymmetric peak. Sufficient cycles of parameter determination are made as are needed for the cessation of a change of the parameter values. The fitting procedure has resulted in reducing the dispersion of

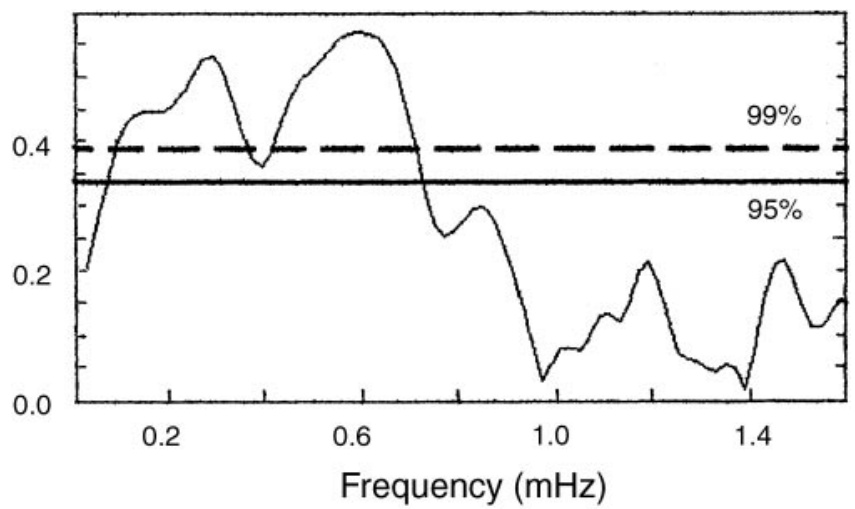

Fig. 3. Coherence spectrum of the filtered microbarographic and seismographic time series shown in Fig. 1. The hypothesis of statistical independence of seismic and ground pressure oscillations from each other is rejected with $95 \%$ and $99 \%$ probability for coherence values above the solid and dashed horizontal lines, respectively. The left maximum corresponds to the $57.1 \mathrm{~min}$ period

the time series $F^{*}\left(t_{i}\right)$ to no more than $6 \%$ of that for the time series $F\left(t_{i}\right)$.

We have utilized this procedure of approximating the function $F(t)$ to obtain the frequencies $\sigma_{j}$ of the ${ }_{0} S_{2}$ multiplet components for a subsequent search of their phases. To derive $\Delta \psi_{m}$ reliably, we have checked phase behavior to avoid any perceptible jumps of phase during our measurements. Time intervals (common to microbarographic and seismographic records) of the duration between $10 \mathrm{~h}$ and $30 \mathrm{~h}$ with a high signal-to-noise ratio and without the phase jumps have been found for each detected ${ }_{0} S_{2}$ component. (See procedure details in Savina et al., 1985.) The oscillation phases in these intervals have been analyzed. The comparison of $\Delta \psi_{m}$ has been led to a surprising conclusion:

$\left|\Delta \psi_{m>0}-\Delta \psi_{m<0}\right|=\pi$

taking into account an experimental error (Table 1).

\section{Model of global atmospheric oscillations}

The model of atmospheric oscillations, forced by seismic movements should be considered to understand the observational inference in Eq. (2). Following Garmash et al. (1989), we present a brief description of a simple model for steady global atmospheric oscillations of the periods considered. The atmosphere is considered as a layer of small thickness compared with the radius of the Earth $(a)$. The background atmosphere is assumed to be isothermal and at rest. Thus, the basic density $(\bar{\rho})$ and pressure $(\bar{p})$ are proportional to $\exp (-z / H)$, where $z$ is the height, and $H$ is the scale height. The gravitational potential perturbation $(\Omega)$, induced by seismic oscillations, is assumed to be independent of $z$. The atmospheric oscillations are described by a set of the following linear equations neglecting of both dissipative processes and diurnal rotation: 
$\frac{\partial u}{\partial t}=-\frac{1}{a} \frac{\partial}{\partial \vartheta}\left(\frac{p^{\prime}}{\bar{\rho}}+\Omega\right)$

$\frac{\partial v}{\partial t}=-\frac{1}{a \sin \vartheta} \frac{\partial}{\partial \varphi}\left(\frac{p^{\prime}}{\bar{\rho}}+\Omega\right)$

$\frac{\partial w}{\partial t}=-\frac{1}{\bar{\rho}} \frac{\partial p^{\prime}}{\partial z}-g \frac{\rho^{\prime}}{\bar{\rho}}$

$\frac{\partial \rho^{\prime}}{\partial t}-\frac{w \bar{\rho}}{H}=-\bar{\rho} \chi$

$\frac{\partial p^{\prime}}{\partial t}-\frac{w \bar{p}}{H}=\gamma g H\left(\frac{\partial \rho^{\prime}}{\partial t}-\frac{w \bar{\rho}}{H}\right)$

where $u, v$, and $w$ are the respective $\vartheta, \varphi$, and $z$ components of the velocity $\boldsymbol{V} ; \rho^{\prime}$ and $p^{\prime}$ are the density and pressure perturbations, respectively; $g$ is the acceleration of gravity; $\gamma$ is the ratio of the specific heat capacity at constant pressure to that at constant volume; $\chi \equiv \operatorname{div} \boldsymbol{V}$. (The effect of the Earth's daily rotation is briefly considered in Sect. 5).

For a steady periodic process any dependent variable $(f)$ is written

$f(t, \vartheta, \varphi, z)=f_{\sigma}(\vartheta, \varphi, z) e^{i \sigma t}$

with $\sigma$ the oscillation frequency. Using Eq. (4) in Eq. (3) we have

$p_{\sigma}^{\prime}=\frac{i g \bar{\rho}}{\sigma}\left(\gamma H \chi_{\sigma}-w_{\sigma}\right)$

and

$$
\begin{aligned}
\left(\frac{\sigma^{2}}{g}+\frac{g}{\sigma^{2}} \Delta_{\vartheta, \varphi}\right) w_{\sigma}= & \gamma H\left(\frac{1}{H}-\frac{\partial}{\partial z}+\frac{g}{\sigma^{2}} \Delta_{\vartheta, \varphi}\right) \chi_{\sigma} \\
& -\frac{i}{\sigma} \Delta_{\vartheta, \varphi} \Omega_{\sigma}
\end{aligned}
$$

where $\Delta_{\vartheta, \varphi}$ is the horizontal Laplace operator. The Eqs. (3) are reduced to a single equation for $\chi_{\sigma}$ alone:

$$
\begin{aligned}
& \left(H \frac{\partial^{2}}{\partial z^{2}}-\frac{\partial}{\partial z}+\frac{\sigma^{2}}{\gamma g}\right) \chi_{\sigma} \\
& \quad=\frac{g}{\sigma^{2}} \Delta_{\vartheta, \varphi}\left[\left(\frac{\gamma-1}{\gamma}-\frac{\sigma^{2} H}{g}\right) \chi_{\sigma}+\frac{i \sigma^{3}}{\gamma g^{2}} \Omega_{\sigma}\right] .
\end{aligned}
$$

Since a spherical surface function $Y_{n}^{m}(\vartheta, \varphi)$ is the solution of the equation

$a^{2} \Delta_{\vartheta, \varphi} Y_{n}^{m}+n(n+1) Y_{n}^{m}=0$,

any $f_{\sigma}$ is written

$f_{\sigma}(z, \vartheta, \varphi)=\sum_{n, m} f_{\sigma, n}^{m}(z) Y_{n}^{m}(\vartheta, \varphi)$.

Following the common practice of changing variables,

$x=\frac{z}{H}$

and

$y_{\sigma, n}^{m}=\chi_{\sigma, n}^{m} e^{-\frac{x}{2}}$, the problem of global atmospheric oscillations is reduced to solving the vertical structure equation:

$\frac{\mathrm{d}^{2} y_{\sigma, n}^{m}}{\mathrm{~d} x^{2}}-\lambda_{\sigma, n}^{2} y_{\sigma, n}^{m}=\beta_{\sigma, n}^{m} e^{-\frac{x}{2}}$,

where

$\lambda_{\sigma, n}^{2}=\frac{1}{4}-\frac{(\gamma-1) H}{\gamma h_{\sigma, n}}+C_{\sigma}\left(\frac{H}{h_{\sigma, n}}-\frac{1}{\gamma}\right)$,

$\beta_{\sigma, n}^{m}=-\frac{i \sigma^{3} H}{\gamma g^{2} h_{\sigma, n}} \Omega_{\sigma, n}^{m}$,

$h_{\sigma, n}=\frac{a^{2} \sigma^{2}}{n(n+1) g}$,

$C_{\sigma}=\frac{H \sigma^{2}}{g}$.

The upper boundary condition Eq. (12) is traditional, requiring the finiteness of $y_{\sigma, n}^{m}(x)$ as $x \rightarrow \infty$. The lower boundary condition at $x=0$, as for $\Omega$, incorporates forcing atmospheric oscillations by seismic oscillations through the piston mechanism:

$w_{\sigma, n}^{m}(0)=i \sigma \Delta a_{\sigma, n}^{m}$,

where $\Delta a_{\sigma, n}^{m}$ is the amplitude of ground displacement for the oscillation mode $(\sigma, n m)$, and $w_{\sigma, n}^{m}$ may be written from Eq. (6) as

$w_{\sigma, n}^{m}=\frac{\gamma}{\frac{1}{h_{\sigma, n}}-\frac{\sigma^{2}}{g}}\left[e^{\frac{x}{2}}\left(\frac{\mathrm{d}}{\mathrm{d} x}+\frac{H}{h_{\sigma, n}}-\frac{1}{2}\right) y_{\sigma, n}^{m}+\frac{\beta_{\sigma, n}^{m}}{C_{\sigma}}\right]$.

Finally, the solution of the Eq. (12) is

$$
\begin{aligned}
y_{\sigma, n}^{m}= & \frac{\beta_{\sigma, n}^{m} e^{-\frac{x}{2}}}{\frac{1}{4}-\lambda_{\sigma, n}^{2}}+\frac{e^{-\lambda_{\sigma, n} x}}{\frac{H}{h_{\sigma, n}}-\lambda_{\sigma, n}-\frac{1}{2}} \\
& \times\left\{\frac{i \sigma}{\gamma}\left(\frac{1}{h_{\sigma, n}}-\frac{\sigma^{2}}{g}\right) \Delta a_{\sigma, n}^{m}+\beta_{\sigma, n}^{m}\left[\frac{1-\frac{H}{h_{\sigma, n}}}{\frac{1}{4}-\lambda_{\sigma, n}^{2}}-\frac{1}{C_{\sigma}}\right]\right\} .
\end{aligned}
$$

Using Eq. (5), we also give the expression for the pressure perturbation, which will be used in the discussion:

$$
\begin{aligned}
\frac{p_{\sigma, n}^{\prime m}}{\bar{p}}=\frac{\Delta a_{\sigma, n}^{m}}{H}\left[1-\frac{H}{\frac{H}{h_{\sigma, n}}-\lambda_{\sigma, n}-\frac{1}{2}}\left(\frac{1}{h_{\sigma, n}}-\frac{\sigma^{2}}{g}\right)\right] e^{\left(\frac{1}{2}-\lambda_{\sigma, n}\right) x} \\
+\frac{C_{\sigma} \Omega_{\sigma, n}^{m}}{g h_{\sigma, n}}\left\{\frac{1}{\frac{1}{4}-\lambda_{\sigma, n}^{2}}+\frac{e^{\left(\frac{1}{2}-\lambda_{\sigma, n}\right) x}}{\frac{H}{h_{\sigma, n}}-\lambda_{\sigma, n}-\frac{1}{2}}\left[\frac{1-\frac{H}{h_{\sigma, n}}}{\frac{1}{4}-\lambda_{\sigma, n}^{2}}-\frac{1}{C_{\sigma}}\right]\right. \\
\left.-\frac{\frac{1}{H}\left[e^{\left(\frac{1}{2}-\lambda_{\sigma, n}\right) x}-1\right]}{\frac{1}{h_{\sigma, n}}-\frac{\sigma^{2}}{g}}\left[\frac{1-\frac{H}{h_{\sigma, n}}}{\frac{1}{4}-\lambda_{\sigma, n}^{2}}-\frac{1}{C_{\sigma}}\right]\right\} .
\end{aligned}
$$


The first and second terms of Eq. (20) describe piston and gravitational forcings of atmospheric oscillations, respectively.

\section{Discussion}

The detection of periodicity at $54 \mathrm{~min}$ in auroral bursts by Roldugin (1967) may be considered as the first evidence of the ${ }_{0} S_{2}$ mode penetrating into the atmosphere. Later Bobova et al. (1990) have revealed all five components of the ${ }_{0} S_{2}$ multiplet in the spectrum of the 1-min geomagnetic AE-index for the 14-year period. Both results may be interpreted in terms of the ionospheric wind dynamo (e.g., Kelley, 1989). That is when the wind 54-min variation in the lower thermosphere generates a corresponding current variation which leads to resultant periodicity at $54 \mathrm{~min}$ in electric fields and magnetic perturbations. Other observational evidence of the Earth's ${ }_{0} S_{2}$ mode penetrating into the atmosphere consists of revealing steady prominent features near the ${ }_{0} S_{2}$ multiplet frequencies in spectra of ground pressure variations (Garmash et al., 1989; Lin'kov et al., 1991). The relation between seismic and atmospheric oscillations is confirmed, e.g. by deriving the coherence coefficient of 0.72 (at the $95 \%$ confidence level) at the 55 min period from synchronous microbarographic and seismographic runs in St. Petersburg $\left(60^{\circ} \mathrm{N}, 30^{\circ} \mathrm{E}\right)$ in $1981-82$ (Garmash et al., 1989).

Garmash et al. (1989) have put forward the piston mechanism for the explanation of long-period Earth oscillations penetrating into the atmosphere. The penetrating can occur at the resonant frequencies determined by the condition

$h_{\sigma, n}=\gamma H$

for free oscillations of the atmosphere (Dikii, 1965). This condition provides

$\frac{H}{h_{\sigma, n}}-\lambda_{\sigma, n}-\frac{1}{2}=0$.

Conceivably the piston mechanism of the condition (21) is realized for some Earth oscillations penetrating into the atmosphere (Petrova et al., 1996). This mechanism is unlikely suited for the generation of atmospheric oscillations by the Earth's ${ }_{0} S_{2}$ mode. The point is that the horizontal structure of the ${ }_{0} S_{2}$ mode corresponds to the $Y_{n}^{m}(\vartheta, \varphi)$ functions with $n=2$, whereas near the 54 min period the condition (21) provides penetration of oscillations with $n=35-40$.

However, there is another solution in the free oscillation theory for the atmosphere (Dikii, 1965):

$h_{\sigma, n}=\frac{g}{\sigma^{2}}$.

Following Dikii (1965), we name this solution the Pekeris solution. Substitution of Eq. (15) into Eq. (23) determines Pekeris' frequencies:

$\sigma_{P, n}=\sqrt[4]{n(n+1)} \sqrt{\frac{g}{a}}$
The expression (20) shows the resonance amplification of $p_{\sigma, n}^{\prime m}$ for $x>0$ near the frequencies $\sigma_{P, n}$ due to gravitational potential perturbation. We should mention in passing that Dikii (1965) considered free atmospheric oscillations at $\sigma_{P, n}$ as physically unrealizable ones.

Garmash et al. (1989) called attention to the following surprising coincidences for the second Pekeris mode $(n=2)$ and the Earth's ${ }_{0} S_{2}$ mode: (1) the period $\tau_{P, 2} \approx 54 \mathrm{~min}$ is practically equal to $\tau$ of the central component $(m=0)$ of the ${ }_{0} S_{2}$ multiplet; (2) their horizontal structures agree as described by $Y_{2}^{m}(\vartheta, \varphi)$. It is these coincidences that point toward a way to explain the ${ }_{0} S_{2}$ mode penetrating into the atmosphere. Unfortunately, the simple oscillation model considered is not able to provide any quantitative treatment of penetration. Later we will consider this question in detail.

The coincidental points specified also suggest explanation of the phase relationship Eq. (2). The explanation is based on the following:

A. The gravitational potential perturbation and ground displacement result from common seismic processes. Therefore,

$\Omega_{\sigma, n}^{m}=c \Delta a_{\sigma, n}^{m}$

with $c$ the unknown complex number. A simple example of the $c$ expression was derived by Garmash et al. (1989) on the assumption that $\Omega$ is induced by small vertical displacements of the Earth's surface neglecting Earth's density variations:

$c=\frac{3 g}{2 n+1}\left(1+\frac{z}{a}\right)^{-(n+1)}$

with $g$ the acceleration of gravity at the surface.

B. Relative contributions of ground displacement and gravitational acceleration perturbation to the acceleration, recorded by a seismograph, are unknown. However, from Eq. (25), the recorded acceleration is linear in the ground displacement with some certain complex factor.

C. The expression (20) for the pressure perturbation contains terms with both the factor of $\left(1 / h_{\sigma, n}-\sigma^{2} / g\right)$ and its inverse.

D. The expression $\left(1 / h_{\sigma, n}-\sigma^{2} / g\right)$ changes its sign at passage of $\sigma$ through $\sigma_{P, n}$. In the case under consideration that is easily seen from the expression

$\frac{1}{h_{\sigma, 2}}-\frac{\sigma^{2}}{g} \simeq-\frac{4 \sqrt{6}}{a} \frac{\delta \sigma}{\sigma_{P, 2}}$

obtained for $|\delta \sigma| \ll \sigma_{P, 2}$ where $\delta \sigma=\sigma-\sigma_{P, 2}$.

Thus, one might therefore expect that $p_{\sigma .2}^{\prime m}$ changes sign when going from $\sigma_{m<0}$ to $\sigma_{m>0}$. This sign change must be revealed in observations as the phase relationship Eq. (2). The relationship may be interpreted as an observational proof of global atmospheric oscillations associated with the second Pekeris mode. It should be noted that the relationship (2) can be explained only under the assumption of forcing atmospheric oscillations by the Earth's ${ }_{0} S_{2}$ oscillations. The fulfilment of 
the relationship is therefore a further proof of the ${ }_{0} S_{2}$ mode penetrating into the atmosphere.

It should be stressed that the simple model of Sect. 4 gives only a general idea of the explanation of the relationship (2). The relationship cannot be derived with this model, as the model is not suited to estimate properly the efficiency of forcing atmospheric oscillations by the Earth's ${ }_{0} S_{2}$ oscillations. That is easily seen if $p_{\sigma, 2}^{\prime m}$ is considered in the vicinity of $\sigma_{P, 2}$ for $|\delta \sigma| / \sigma_{P, 2}$ of no more than the value of $6 \cdot 10^{-2}$, corresponding to the frequency interval of the ${ }_{0} S_{2}$ multiplet (between the components with $m=-2$ and 2). Taking into account in addition, the fact that $C_{\sigma} \ll 1$ for $\sigma=\sigma_{P, 2}$ $\left(C_{\sigma} \approx 3 \cdot 10^{-3}\right)$, the formula (20) may be approximated by the expression

$$
\begin{aligned}
\frac{p_{\sigma, 2}^{\prime m}}{\bar{p}} \simeq & \frac{\Delta a_{\sigma, 2}^{m}}{H}\left[1-\frac{4 \sqrt{6} H}{a} \frac{\delta \sigma_{m}}{\sigma_{P, 2}}\right] \\
& +\frac{\sqrt{6} \Omega_{\sigma, 2}^{m}}{a g}\left\{1+x\left[\frac{\sqrt{6} H}{2 a}\left(\frac{\delta \sigma_{m}}{\sigma_{P, 2}}\right)^{-1}+\frac{1}{\gamma}-1\right]\right\} .
\end{aligned}
$$

We use also

$\eta=\frac{\left|p_{\sigma, n}^{\prime m}\right|}{\Delta a_{\sigma, n}^{m}}$

as an efficiency coefficient for forcing atmospheric oscillations by seismic oscillations (Garmash et al., 1989).

The "piston" term of Eq. (28) does not change its sign at passage of $\sigma$ through $\sigma_{P, 2}$, since

$\frac{4 \sqrt{6} H}{a} \frac{|\delta \sigma|}{\sigma_{P, 2}} \ll 1$.

In the case of the piston mechanism for the ${ }_{0} S_{2}$ mode then:

$\eta=\frac{\bar{p}}{H}$.

This small $\eta$ makes it impossible to detect any effect of the mechanism using the microbarographs (Garmash et al., 1989).

As to the gravitational term of Eq. (28), the resonance appears to be very "sharp" to the extent that it can provide penetration into the atmosphere only for the central component $(m=0)$ of the ${ }_{0} S_{2}$ multiplet. Using, for example, $c$ from Eq. (26) for $z=0$ and $n=2$,

$\eta=\frac{3 \sqrt{6} \bar{p}}{5 a}\left|1-x\left(1-\frac{1}{\gamma}\right)\right|$

outside the resonance peak. Beyond this peak, the efficiency of gravitational transmission of the ${ }_{0} S_{2}$ components into the atmosphere is much less than that by the piston mechanism because of $H \ll a$. There is little likelihood that taking account of the Earth's density variations in $\Omega$ can enhance the gravitational $\eta$ by $2-3$ orders which are required to equalize the contributions of both mechanisms to $\eta$.
Now we consider whether the inferences derived here are changed by introducing the Earth's daily rotation to the model of global atmospheric oscillations. The rotation splits frequencies of free atmospheric oscillations (e.g., Dikii, 1965). We have estimated the order-ofmagnitude of the splitting based on the simple linear model of global oscillations at the limit of large $\sigma / 2 \omega$ where $\omega$ is the angular velocity of Earth (see Dikii's formula, 2.8). For free atmospheric oscillations described with using the functions $Y_{n}^{m}(\vartheta, \varphi)$ the rotational perturbation of the frequency is

$\Delta \sigma_{n}^{m}=\frac{m \omega}{n(n+1)}$,

$-n \leq m \leq n$. For the second atmospheric Pekeris mode the frequency spacing between neighboring multiplet components is $\omega / 6=1.93 \mu \mathrm{Hz}$. That for the ${ }_{0} S_{2}$ multiplet is $4.5-4.8 \mu \mathrm{Hz}$. Taking into account that the $m$-component of the ${ }_{0} S_{2}$ mode penetrates into the atmosphere by forcing atmospheric oscillations in the like $m$-component of the Pekeris mode two conclusions can be made: (1) the spacing between the ${ }_{0} S_{2}$ components is more than double that between the Pekeris components. When passing from $\sigma_{m<0}$ to $\sigma_{m>0}$ the sign change in $p_{\sigma, 2}^{\prime m}$ is therefore provided, and the explanation of the phase relationship Eq. (2) retains its validity correspondingly; (2) due to the Earth's rotation the ${ }_{0} S_{2}$ multiplet frequencies turn out closer to the respective resonant frequencies of the Pekeris mode. This fact is capable of increasing the efficiency of gravitational transmission of the ${ }_{0} S_{2}$ components into the atmosphere.

Thus the simple model considered does not provide the ultimate answer what mechanism forces atmospheric oscillations at the frequencies of the ${ }_{0} S_{2}$ multiplet. There is some preference of the gravitational mechanism, owing to the "sharp" resonance. However, it may turn out in reality that the piston mechanism contributes as well. Further progression the problem may be made by introducing the following improvements into the model:

A. The expression for $\Omega$ should take into account the Earth's density variations.

B. The Earth's rotation, dissipative processes, and real fields of temperature and wind should be incorporated into the atmospheric oscillation model.

C. The main thing that should be done is to turn to the problem of unsteady oscillations. As a source of forcing, the Earth's ${ }_{0} S_{2}$ oscillations are variable, being completely damped out from time to time. Therefore, the steady oscillation of the simple model is unlikely to be realized.

\section{Conclusion}

The synchronous collocated microbarograph and seismograph measurements have resulted in the phase relationship Eq. (2) for the ${ }_{0} S_{2}$ multiplet components with different signs of the number $m$. For the first time the second Pekeris mode of global atmospheric oscilla- 
tions has been revealed experimentally. The relationship should also be read as a further proof of penetration of the Earth's ${ }_{0} S_{2}$ mode into the atmosphere. The linear non-dissipative model of steady forced oscillations in isothermal atmosphere at rest does not describe penetration of the ${ }_{0} S_{2}$ mode into the atmosphere adequately.

Acknowledgements. Our work was supported by the Russian Foundation for Basic Research under grant 93-05-8210 and by the "Russian Universities - Basic Research" program under project 1-2234. We are grateful to the anonymous referees and M.L. Pudovkin for very helpful comments on the manuscript.

Topical Editor F. Vial thanks J.M. Forbes and A.D. Richmond for their help in evaluating this paper.

\section{References}

Aki, K., and P. Richards, Quantitative seismology. Theory and methods, vol 1, W. H. Freeman, San Francisco, 1980.

Bobova, V. P., K. S. Osypov, N. G. Savina, B. M. Vladimirsky, and M. I. Pudovkin, Probability of the seismic origin of long-period $(\mathrm{T}=1-4 \mathrm{~h})$ variations of geomagnetic disturbances, Geomagn. Aeron., 30, 492-494, 1990.

Buland, R., J. Berger, and F. Gilbert, Observations from the IDA network of attenuation and splitting during a recent earthquake, Nature, 277, 358-362, 1979.

Dikii, L. A., The terrestrial atmosphere as an oscillating system. Izvest. Atmos. Ocean. Phys., 1, 275-286, 1965.

Garmash, S. V., Y. M. Lin'kov, L. N. Petrova, and G. M. Shved, Generation of atmospheric oscillations by seismic-gravity oscillations of the Earth, Izvest. Atmos. Ocean. Phys., 25, 952959, 1989.

Kelley, M. C., The Earth's ionosphere: plasma physics and electrodynamics, Academic Press, San Diego, 1989.
Kozhevnikova, E. G., E. G. Orlov, and Y. M. Lin'kov, Pressure variations as a noise for long-period observations, in Problems of geophysics, Eds. Molochnov G. V., and A. S. Semenov, vol 28, Leningrad University Press, Leningrad, 229-233, 1980.

Lin'kov, Y. M., L. N. Petrova, and D. D. Zuroshvili, Seismogravitational oscillations of the Earth and associated disturbances of the atmosphere, Trans. USSR Acad. Sci: Earth Sci., 306, 13-16, 1991.

Lin'kov, Y. M., S. Ya. Tipisev, and V. V. Butsenko, Noise-protected long-period seismograph and analysis of its records, in Geophysical apparatus, Ed. Matveev, A. V., vol 75, Nedra, Leningrad, 78-87, 1982.

Mendiguren, J., Identification of free oscillation spectral peaks for 1970 July 31, Colombian deep shock using the excitation criterion, Geophys. J. R. Astron. Soc. 33, 281-321, 1973.

Petrova, L. N., K. S. Osypov, D. D. Savel'ev, and G. M. Shved, Forcing atmospheric oscillations by long-period seismic oscillations: a case study, J. Atmos. Terr. Phys. 58, 1317-1322, 1996.

Roldugin, V. K., Recurrence of aurora outbursts, Geomagn. Aeron., 7, 1116-1117, 1967.

Savarensky, E. F., and O. P. Kirnos An introduction to seismology and seismometry, GITTL, Moscow, 1955.

Savina, N. G., L. N. Petrova, and T. B. Yanovskaya, Analysis of the long-period part of the spectrum of free oscillations of the Earth, in Computational seismology, Eds. Keilis-Borok V. I., and A. L. Levshin, 15, Allerton Press, 136-144, 1985.

Stein, S., and R. Geller, Amplitudes of the Earth's split normal modes, J. Phys. Earth, 25, 117-142, 1977.

Suda, N., K. Nawa, and Y. Fukao, Earth's background free oscillations, Science, 279, 2089-2091, 1998.

Tanimoto, T., J. Um, K. Nishida, and N. Kobayashi, Earth's continuous oscillations observed on seismically quiet days, Geophys. Res. Lett., 25, 1553-1556, 1998. 